\title{
Energy Model for a Differential Guide Mobile Robot using Modelica Language
}

\section{Fadlo Said $^{\mathrm{a}}$, Rabbah Nabila ${ }^{\mathrm{b}}$, Ait elmahjoub Hafid ${ }^{\mathrm{c}}$}

${ }^{a}$ Dept.Structural Engineering, Intelligent Systems, and Electrical Energy, Ensam, Universite Hassan II, Casablanca, Morocco. E-mail: fadlosaid@gmail.com

${ }^{b}$ Dept .Structural Engineering, Intelligent Systems, and Electrical Energy, Ensam, Universite Hassan II, Casablanca, Morocco. E-mail: nabila_rabbah@yahoo.fr

${ }^{\mathrm{c} D e p t . S t r u c t u r a l ~ E n g i n e e r i n g, ~ I n t e l l i g e n t ~ S y s t e m s, ~ a n d ~ E l e c t r i c a l ~ E n e r g y, ~ E n s a m, ~ U n i v e r s i t e ~ H a s s a n ~ I I, ~}$ Casablanca, Morocco. E-mail: aitelmahjoub@gmail.com

Article History: Received: 11 January 2021; Accepted: 27 February 2021; Published online: 5 April 2021

\begin{abstract}
Energy consumption is an important element in the autonomy of mobile robots. In this paper, an energy model has been developed in Modelica language. The power of the Modelica ${ }^{\circledR}$ language is shown by simulating the energy model for a differential guide mobile robot as well as the motors. The model is tested with typical motor energy, mixed energy models, and a trapezoidal velocity as input.
\end{abstract}

Keywords: Energy Model, DC Motor, Modelica Model, Differential Guide Mobile Robot.

\section{Introduction}

Modern mobile robots have many advantages: high- efficiency, reduced cost, customizable, upgradeable. Their main disadvantage is a limited energy resource, because of relatively small capacity of batteries. The abovementioned problem can be solved by increasing battery life or reducing energy consumption. It is well-known that driving motors are a major source of energy consumption [1]-[2]. In those works, an optimization is applied to the motor energy model, to calculate the optimal angular velocities of the wheels, to minimize the energy consumption, for a mobile robot when following a straight path. Despite the researcher's effort to obtain better trajectories [3],[4],[5],[6], the resulting energy values did not reflect how the mobile dynamic parameters contribute, such as weight of the mobile or load weight.

The need to understand and predict the influence of this parameters in functioning and performance of the robot motivates the development of new models. Recently, Morales et al[7] presented an energy model for the differential guide mobile robot. The dynamic mobile robot model and the dynamic motor model are calculated, then the dynamic models are mixed, matching the torque values.

In this present work, the focus is on the development of an energy consumption model for wheeled mobile robots applying modeling and simulation techniques, withModelica language, which is not reported yet by other researchers.

\section{Model Background}

\section{Modeling DC Motor}

The DC motor behaviour is characterized using an equivalent circuit model. Fig 1 shows the DC motor circuit with Torque and Rotor angle consideration. The differential equations that describe the dynamic model are:

$$
\left\{\begin{array}{c}
L \frac{d i}{d t}+R i+K_{\omega} \dot{\theta}=V \\
I_{S} \ddot{\theta}-K_{t} i+v \dot{\theta}+\tau=0
\end{array}\right.
$$

Where:

$\mathrm{V}$ and $\mathrm{i}$ are the armature voltage and current.

$\mathrm{R}$ and $\mathrm{L}$ are the armature resistance and inductance.

$v$ is the viscous friction coefficient.

$\tau$ is the dynamic load applied to the motor.

$K_{t}$ is the motor torque constant.

$K_{\omega}$ is the voltage constant.

$I_{S}$ is the motor shaft inertia. 
$\theta=\left[\theta_{1} \theta_{2}\right]$ are the angular positions of the wheels.

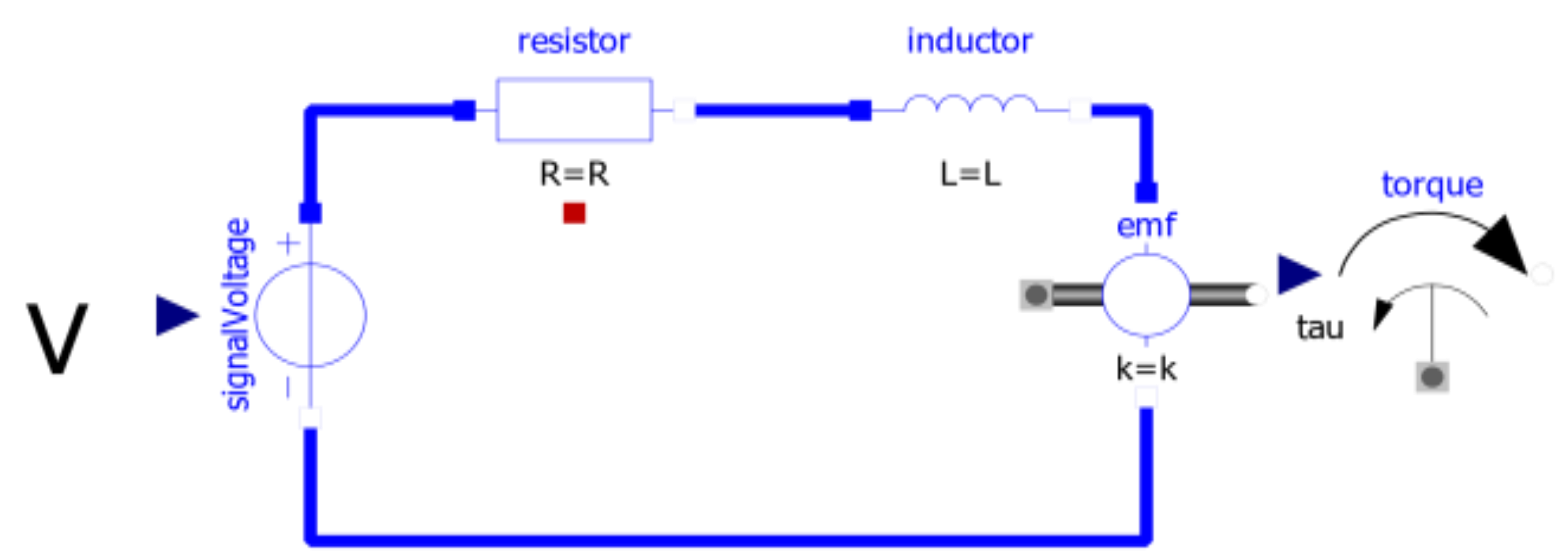

Fig. 1. Schematic Diagram of the DC Motor

The first expression in (1) is the voltage equation for the DC motor, and the second onereflects torque forces applied to the DC motor. As in the Mixed energy model [7], in ourproposed model, the torque value of a DC dynamic motor model is taken intoconsideration. As the electric time constant $\mathrm{L} / \mathrm{R}$ may be neglected, if compared to the mechanical time constant $i / v$. Hence, one may consider $\mathrm{L}=0$, and the first equation yields:

$$
i=\frac{V-K_{\omega} \dot{\theta}}{R}
$$

\section{Mobile Robot Description}

The Pioneer 3-DX with onboard PC is a fully autonomous intelligent mobile robot, the Pioneer 3 is fully capable of mapping its environment, finding its way home and performing other sophisticated path-planning tasks. Weighing only $9 \mathrm{~kg}$ (20 pounds with one battery), the basic Pioneer 3-DX mobile robots are lightweight, but their strong aluminium body and solid construction make them solid[8].

These characteristics also permit them to carry extraordinary payloads: The Pioneer 3-DX can carry upto $23 \mathrm{Kg}$ additional weight. This family of robots is composed of several main parts:

- Deck

- Motor Stop Button

- User Control Panel

- Body, Nose, and Accessory Panels

- Sonar Array(s)

- Motors, Wheels, and Encoders

- Batteries and Power

\section{Model Implementation}

The model as displayed in Fig.1 is implemented in the Modelica environment. The result is displayed in Fig.2.

The structure of the model is:

- The upper branch shows control of DC Motor.

- On the left bottom, you can see the DC motor.

- On the right branch shows an ideal Gear, an ideal rolling wheel, and a mass. It is the robot mobile mass, in figure $7.6 \mathrm{~kg}$. 


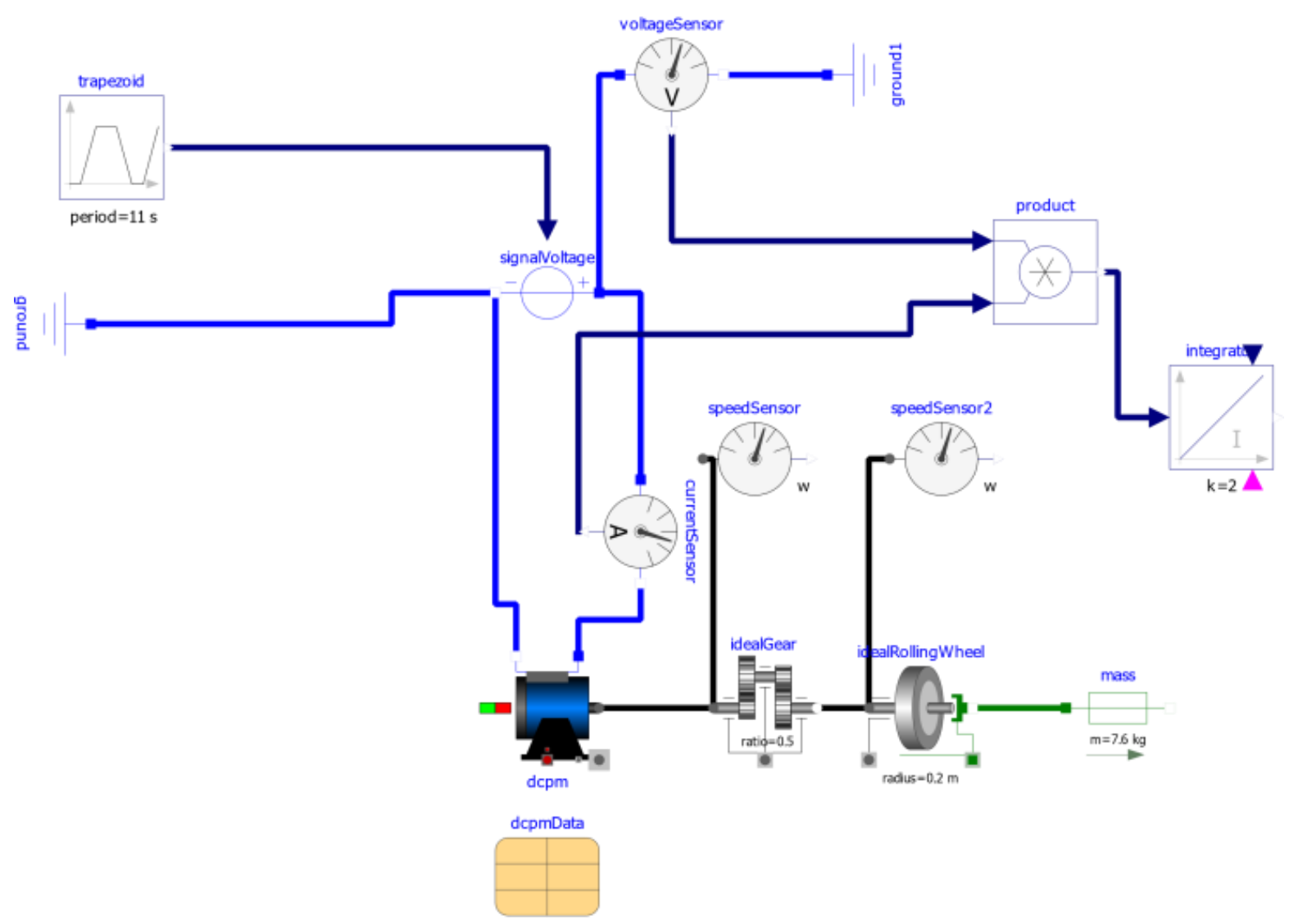

Fig. 2. Model of the mobile robot in Openmodelica environment

\section{Model Results and Disscussion}

To set up the Modelica model represented by Fig.3, the mobile and motor parameters of the P3-DX mobile platform present in Kim's work [1],[2], were used. These parameters are shown in the tables (table I, II).

Tab. 1. Motor parameter of the WMR, P3-DX

\begin{tabular}{llll}
\hline Parameters & \multicolumn{1}{c}{ Values } & Parameters & \multicolumn{1}{c}{ Values } \\
\hline $\mathrm{R}$ & $0.7 \Omega$ & $K_{t}$ & $0.88 \mathrm{Nm} / \mathrm{A}$ \\
$v$ & $0.035 \mathrm{Nm} /(\mathrm{rad} / \mathrm{s})$ & $K_{\omega}$ & $0.88(\mathrm{rad} / \mathrm{s}) / \mathrm{V}$ \\
$I_{S}$ & $0.0713 \mathrm{Kgm}^{2}$ & & \\
\hline
\end{tabular}

Tab. 2. Mobile parameters of the WMR, P3-DX

\begin{tabular}{lccc}
\hline Parameters & Values & Parameters & Values \\
\hline $\mathrm{r}$ & $0.095 \mathrm{~m}$ & $m_{c}$ & $6.04 \mathrm{Kg}$ \\
$\mathrm{b}$ & $0.165 \mathrm{~m}$ & $m_{\omega}$ & $1.48 \mathrm{Kg}$ \\
\hline
\end{tabular}

The model should passe two kinds of tests to be validated. An accumulative error in the mathematical modeling, in the numerical estimation, or in the calibration of the dynamic parameters will lead to failure of those tests.

In the first test, constants voltages were applied, with the same amplitude, as input of the Motors voltages. The result depicted in the Figure 3 shows that the mass's center of the mobile describes a linear movement with the same orientation of the initial condition.

Figures 4 and 5 illustrate that the angular and linear speeds of the robot changed very sharply. In about $0.03 \mathrm{~s}$, the robot attained the maximum speeds respectively $5 \mathrm{rad} / \mathrm{s}$ and $0.55 \mathrm{~m} / \mathrm{s}$. 


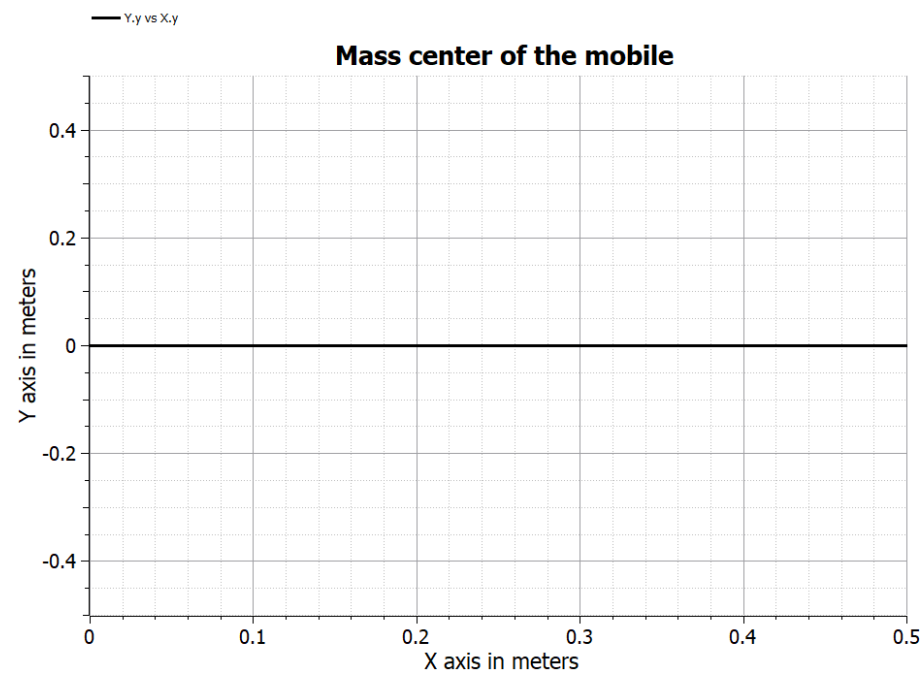

Fig. 3. Response of the model to a constant volatage inputs in both motors

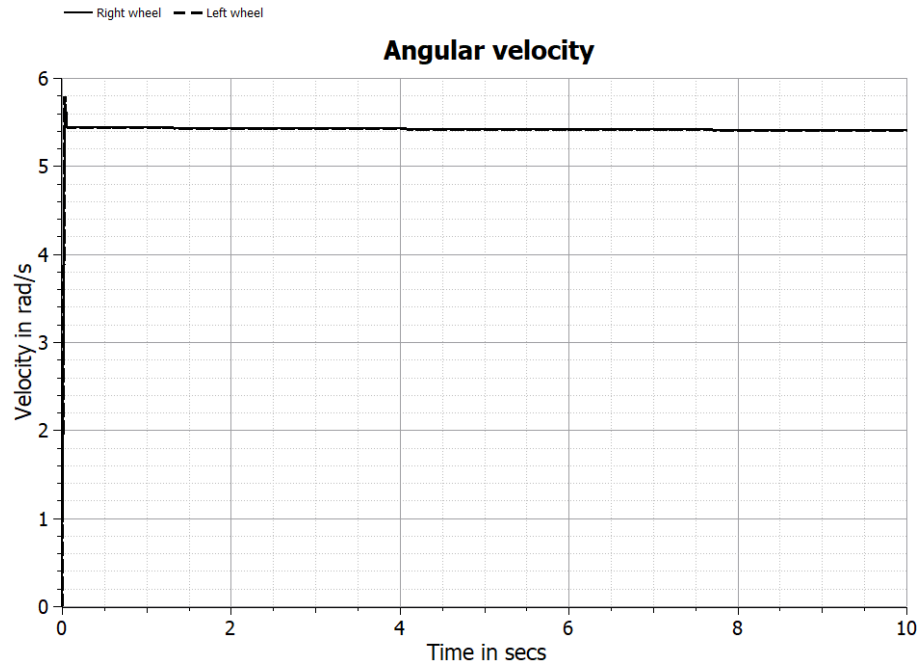

Fig. 4. Angular velocities of the wheels as response ofa constant volatage inputs in both motors

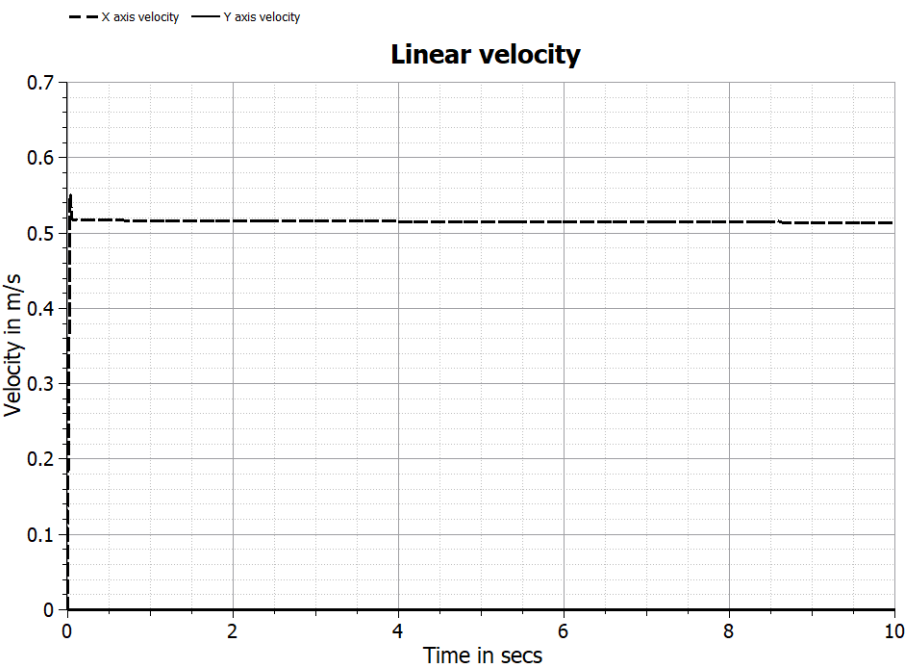

Fig. 5. Linear velocities of the wheels as response ofa constant volatage inputs in both motors

In the second test, just one of the motors was supplied with a constant voltage as an input. In this case, the center mass of the mobile described a circumference with a radius equal to the distance from each of the driving wheels to the symmetry's axis. The results are shown inFigure 6. 


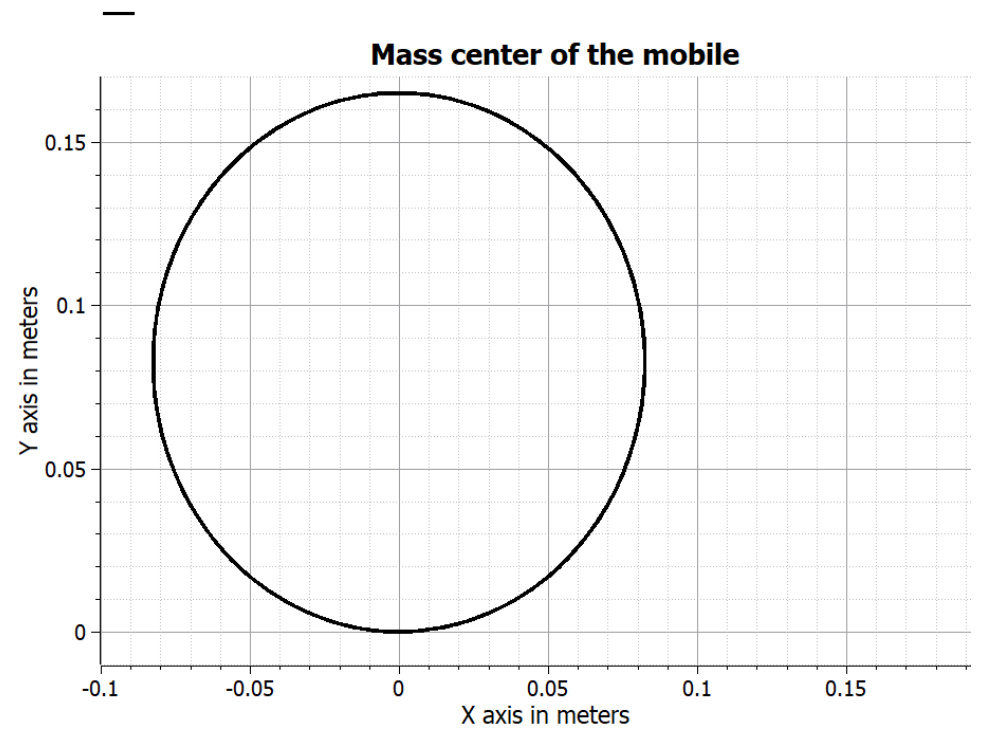

Fig. 6. Response of the model to a constant volatage input to the right motor

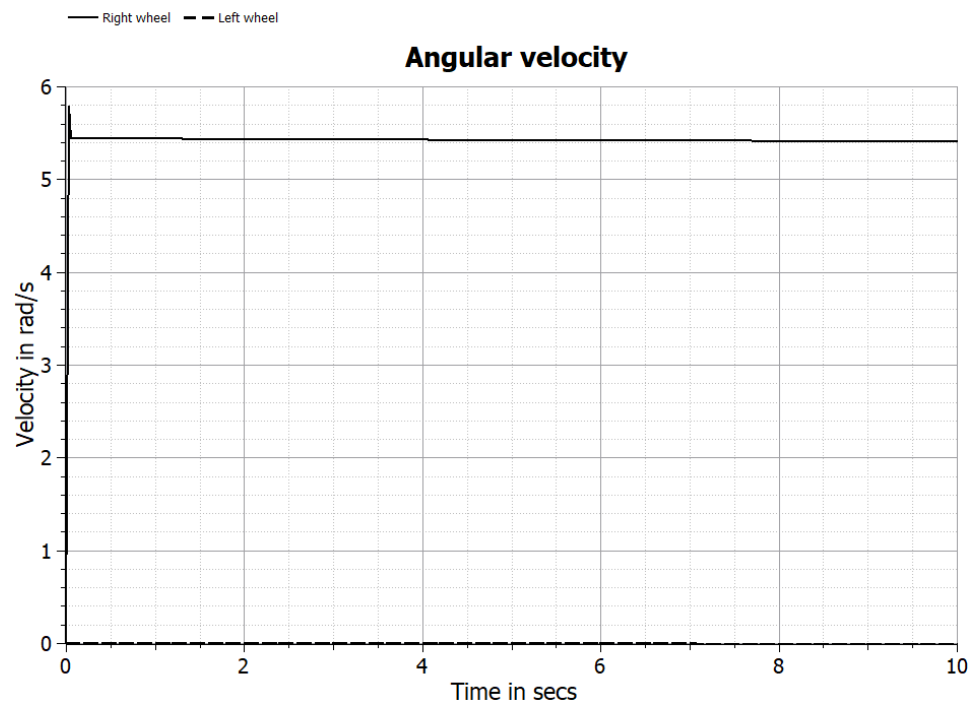

Fig. 7. Angular velocities of the wheels as response ofa constant volatage input to the right motor

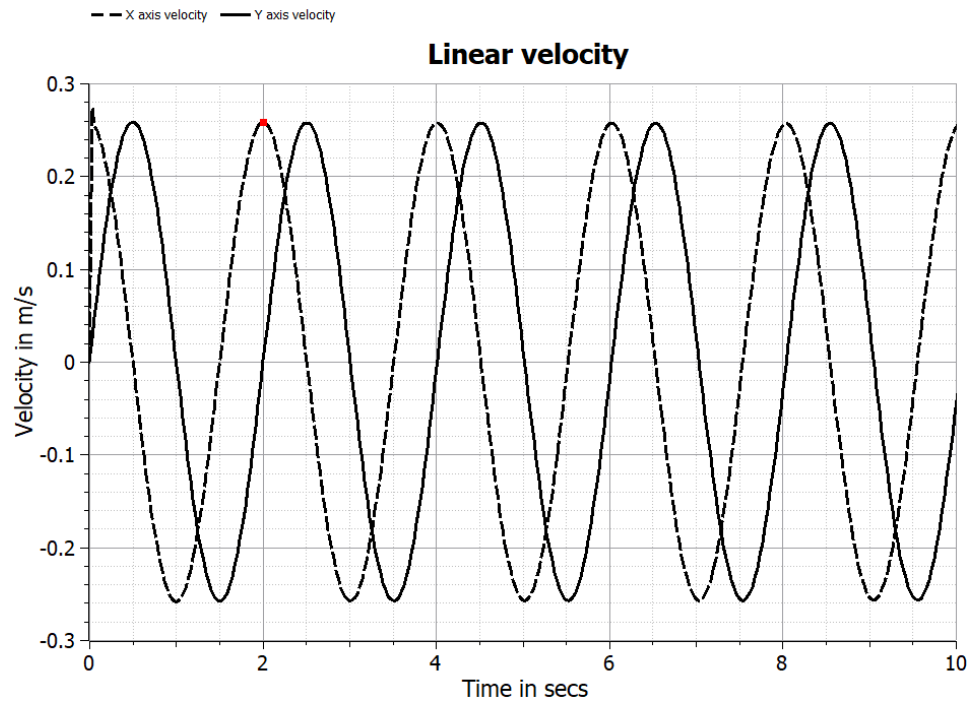

Fig. 5. Linear velocities of the wheels as response ofa constant volatage input to the right motor 
As discussed in the last section, the model has successfully passed the tests. So, we focused on taking advantage of the model to give an estimation of power and energy consumption, with the commonly used trapezoidal velocity profile as the angular velocity of the wheels.

To achieve that, trapezoidal voltages, depicted in Figure 8, were used, with the same amplitude as an input of the motor voltages. As an output, we obtained the angular velocity of the wheels, as shown in Figure 9, with a simulation time of 10 seconds, and initial conditions equal to zero.

Then, the power model can be calculated with the multiplication of the voltage, depicted in Figure 8 , and the current obtained, as shown in figure 10, by the Modelica modelusing equation:

$$
P(t)=V(t) i(t)(3)
$$

Finally, the energy consumption model can be estimated using equation:

$$
E(t)=\int_{0}^{t} P(t) d t(4)
$$

To validate our model, the power and energy consumptioncalculated was compared with the mixed model and the energy model of the engine as shown in Figure 11 and Figure 12.

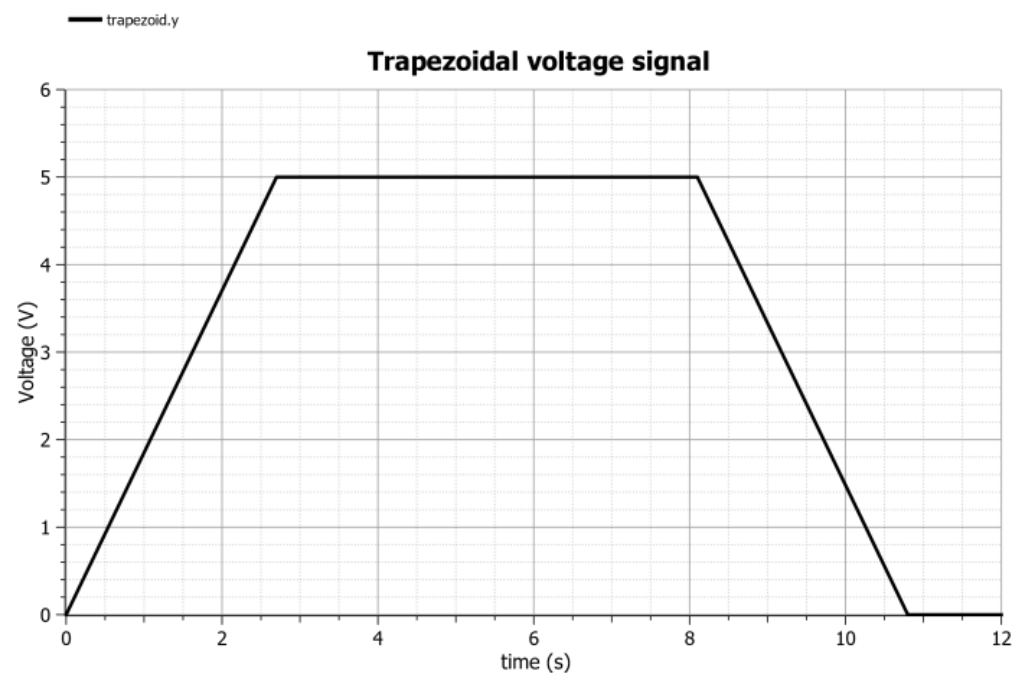

Fig. 8. Trapezoidal input voltage signal to the DC motor

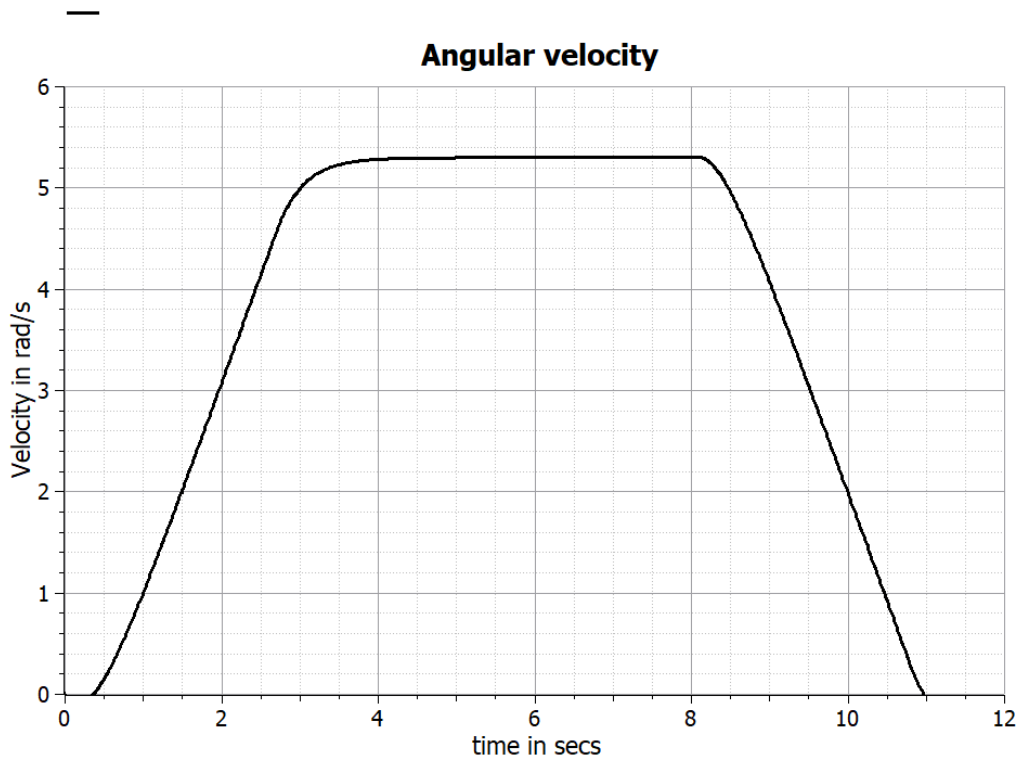

Fig. 9. Angular velocity of the wheel 


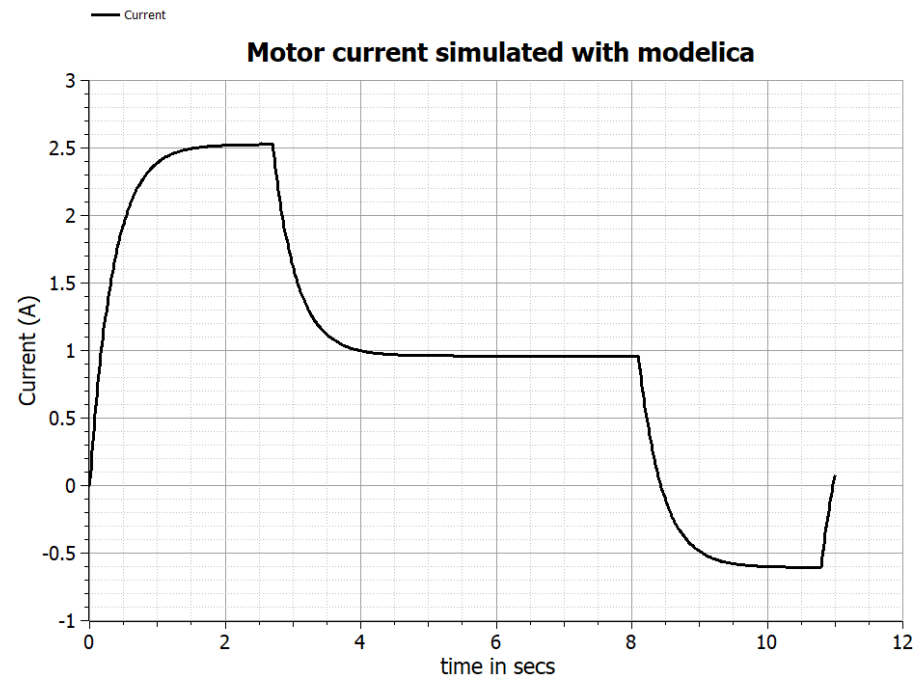

Fig. 10. Moto Current similated with modelica model

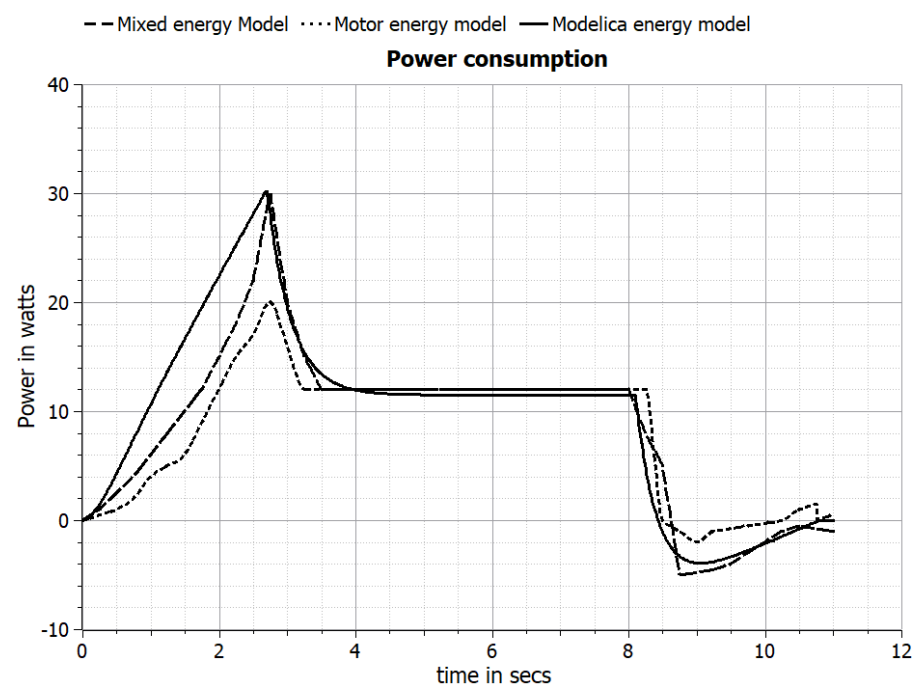

Fig. 11. Power consumption given by differents energy model

Figure 11 shows that, during the acceleration, the power increases faster in the values given by the mixed energy model, and our model show better prediction than the Motor model motor. When the velocity is maximum our model shows that the power decreases slightly. During the deceleration, in the time corresponding to the negative phase of the power consumption, a certain amount of energy is regenerated and can be stored in the batteries.

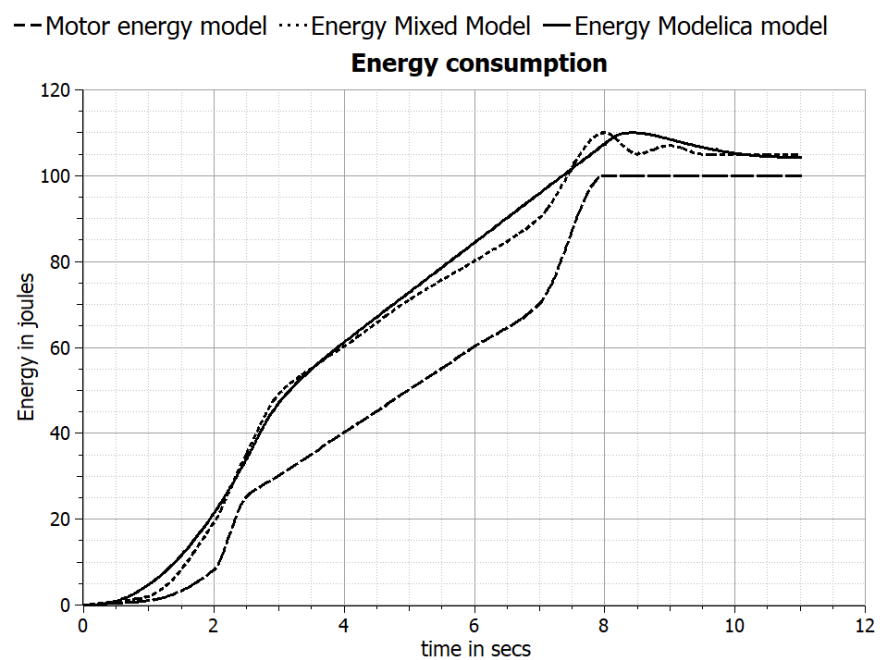

Fig. 12. Energy consumption given by differents energy models 


\section{Conclusion}

In this work, a Model-based on Modelica language for a differential guide robot mobile was performed. An energy model is presented, based on the dynamic motor and the dynamic mobile models, using the motor and mobile parameters of P3-DX robot mobile. The Modelica model was validated. The simulation results show that our model predicts the power consumption during the acceleration and the deceleration phase of robot motion.

\section{References}

1. Kim, H., \& Kim, B.K. (2008). Minimum-energy translational trajectory planning for battery-powered three-wheeled omni-directional mobile robots. In 2008 10th International Conference on Control, Automation, Robotics and Vision, 1730-1735.

2. Tokekar, P., Karnad, N., \& Isler, V. (2014). Energy-optimal trajectory planning for car-like robots. Autonomous Robots, 37(3), 279-300.

3. Liu, S., \& Sun, D. (2011). Optimal motion planning of a mobile robot with minimum energy consumption. In 2011 IEEE/ASME International Conference on Advanced Intelligent Mechatronics (AIM), 43-48.

4. Liu, S., \& Sun, D. (2013). Minimizing energy consumption of wheeled mobile robots via optimal motion planning. IEEE/ASME Transactions on Mechatronics, 19(2), 401-411.

5. Sun, Z., \& Reif, J.H. (2005). On finding energy-minimizing paths on terrains. IEEE Transactions on Robotics, 2l(1), 102-114.

6. Mei, Y., Lu, Y.H., Hu, Y.C., \& Lee, C.G. (2004). Energy-efficient motion planning for mobile robots. In IEEE International Conference on Robotics and Automation, 2004. Proceedings. ICRA'04. 2004, 5, 43444349.

7. Morales, M.F.J., \& Mendoza, J.B.G. (2018). Mixed Energy Model for a Differential Guide Mobile Robot. In 2018 23rd International Conference on Methods \& Models in Automation \& Robotics (MMAR), 114119.

8. Robotics, A. (2003). Pioneer 3TM and Pioneer 2TM H8-Series Operations Manual. ActivMedia Robotics, version, 33-36. 